\title{
Modelo descriptivo de la formación de un huracán
}

Model describing the formation of a hurricane

Diana Maritza Segura Ángel y Sergio Andrés Galindo Torre

Universidad Pedagógica Nacional. dianam071@hotmail.com , galindotorres@hotmail.com

\section{Resumen}

Este proyecto modela la formación de un huracán. Este estudio es de gran importancia debido a que por factores como cambios dimáticos se está incrementando la intensidad de los huracanes y es preciso explicar este fenómeno Debido a que no se encontró ninguna explicación comprensible para llevar al aula sobre la eauación que describe el comportamiento del huracán, se hizo necesario desarrollar estas ecuaciones como la fuerza de Coriolis, la variación de la presión aplicada a una partícula de agua condensada luego se desarrollo un análisis de su comportamiento por medio de la termodinámica; además se realizaron analogías para determinar la variación de la energía que explica el origen de la fuerza del huracán. La implementación de las eauaciones del huracán se realizó en C++. Este no es un modelo predictivo sino descriptivo por esta razón su valor es de carácter pedagógico para la enseñanza pues crea un modelo sencillo para la compresión de fenómenos naturales. Además el proceso de construcción de modelos de nuestro entomo en el aula debe comenzarla y guiarla el Docente.

Este modelo explica la física envuelta en la creación de un huracán e ilustra los principios básicos estudiados en el aula animando a los estudiantes a interesarse por la física por medio de la resolución de problemas observados en la naturaleza.

\section{Palabras claves}

Huracán, modelación, formación

\section{Abstract}

This project is designed to model a physical phenomenon specifically the formation of a hurricane. This study is of great importance due to factors such as global warming and dimate change the number of hurricanes is increasing and we need to explain this phenomenon Because we did not find any pedagogical explanation that describes the behavior of the hurricane, it became necessary to develop these equations based on factors such as the Coriolis force applied to a condensed water partides and analyze their behavior by means of thermodynamics, also analogies were made to determine the variation of the energy that explains its effects. The implementation of the equations of the hurricane was done in $\mathrm{C}++$. This is not a descriptive but predictive model for this reason its value is a teaching for leaming as it creates a simple model for understanding natural phenomena. Besides the construction of models of phenomena of our environment in the dassroom must begin and lead teacher. This model explains the physics involved in the creation of a hurricane and illustrates the basic principles discussed in the dassroom by encouraging students to take an interest in physics through the resolution of problems encountered in nature.

Key Words

Hurricane, formation, modelling 


\section{Introducción}

Cuando se observan los diferentes fenómenos de la naturaleza siempre nos cuestionamos sobre su origen y formación, este trabajo está desarrollado con el objetivo de resolver estas inquietudes de una manera sencilla y didáctica. Creando un modelo con el cual puede interactuar los estudiantes.

La formación de huracanes obedece a diferentes condiciones y es sensible a ellas, es un sistema caótico en el aual no se puede predecir exactamente la trayectoria del huracán, esta es una razón para incentivar a los estudiantes a que se interesen por resolver este tipo de sistemas complejos. El estudio de huracanes se va trabajar en tres partes; condiciones para la formación de un huracán, formación de un huracán en la troposfera y culminaremos con el ojo del huracán y su fuerza.

\section{Modelo}

Variables y fuerzas que contribuyen en la formación del huracán

$>$ Diferencia de presión atmosférica

$>$ Variación temperatura

$>$ Fuerza de Coriolis

$>$ El ojo del huracán

$>$ variación de energía

Diferencia de presión atmosférica

Existen condiciones iníciales para la formación de un huracán como: un mar cálido con una temperatura superior a $27^{\circ} \mathrm{C}$ y un aire húmedo.

El agua se evapora debido al calentamiento del agua por el aumento de temperatura del mar, luego se expande y sube creando una diferencia de presión atmosférica que genera los vientos del huracán (peso del aire por unidad de superficie) haciendo que una corriente de viento se dirija hacia el centro de menor presión (experimentalmente notamos que el gas de mayor temperatura sube y el de menor temperatura baja) debido a la densidad del aire. Cuando las moléculas de gas (vapor de agua) llegan a la troposfera se condensa y por este motivo se libera energía en la atmósfera debido a la variación de temperatura entre las moléculas de gas cálidas y las moléculas condensadas a $75^{\circ} \mathrm{C}$. Según el principio de conservación de la energía, esta energía calorífica (calor latente) produce un calentamiento del aire circundante aumentando la diferencia de presión debido a la densidad de éste que origina vientos.

Este cido se repite debido a que los vientos fluyen hacia las zonas de baja presión, desprendiendo moléculas de gas que liberan energía, este cido se intensifica hasta que los vientos adquieren una gran velocidad

\section{Variación temperatura}

Cuando el huracán inicia su formación con el calentamiento de las moléculas de agua hasta que llegan a la troposfera, la temperatura varia, decrece con la altura a razón de 6.5 C por kilómetro Esta variación se define como gradiente vertical de temperatura. Sin embargo ocurre frecuentemente que se registra un aumento de la temperatura con la altura, debido a que durante la noche la Tierra irradia (pierde calor) y se enfría mucho más rápido que el aire que la circunda; entonces, el aire en contacto con ella será más frío mientras que por encima la temperatura será mayor (inversión de temperatura). En el sistema se estudia la formación del huracán durante el día y La fuerza que actúa sobre el huracán es igual a la variación de la temperatura con la altura (6.5 
o $\mathrm{C}$ por $\mathrm{Km}$.) por una constante igual a la conductividad del aire asociada con la velocidad del huracán, su valor es $k_{1}=3 \times 10^{-24} \mathbf{N}-\mathbf{k m} /{ }^{\circ} \mathrm{C}$. Esta fuerza $\boldsymbol{F}_{\boldsymbol{T}}$ sólo actúa en el eje z

$$
\vec{F}_{T}=-k_{1} \nabla T \hat{k}=\left(6.5^{\circ} C . / \mathrm{km}\right) k_{1} \hat{k}
$$

La constante $k_{1}$ se obtuvo ajustándola para obtener el movimiento "circular" o huracanado de la molécula de agua..

Fuerza de Coriolis

La fuerza de Coriolis desvía el movimiento de un auerpo hacia el occidente u oriente dependiendo de la dirección de la velocidad. Este efecto se debe a la rotación de la Tiena.

La fuerza de Coriolis dirige la rotación de los vientos alrededor de un sistema de baja presión que no permite que el aire pueda ser dirigido hacia el centro de éste, luego el aire es arremolinado alrededor del centro. Los vientos giran en una dirección cidónica. (El término cidónico significa que las moléculas de agua rotan en la misma dirección que lo hace la Tienra debajo suyo)

$$
\vec{F}_{\text {Co }}=-2 m(\vec{\omega} \times \vec{V})
$$

La fuerza de Coriolis ejercida sobre la partícula de agua es perpendicular al eje de rotación de la Tierra y a la velocidad de ésta, por lo tanto varía según la latitud; por ejemplo en el hemisferio norte $\omega$ sale del suelo haciendo que la fuerza de Coriolis desvíe la masa de aire hacia la derecha de su dirección de movimiento (en contra de las manecillas del reloj) alrededor del centro de baja presión, la fuerza de Coriolis tiene sentido opuesto en el hemisferio sur y es nula en el Ecuador en donde $\omega$ es horizontal.

La fuerza desviadora de Coriolis sólo afecta la dirección, no a la velocidad, y tiene una acción mayor cuanto mayor sea la velocidad del huracán velocidad.

En nuestro sistema la fuerza de Coriolis esta dirigida hacia el occidente y depende de la velocidad angular de la tienra y de la velocidad del huracán que a su vez depende del ángulo $\theta$ (latitud) y de la masa de la molécula de agua.

\section{Direcciones en que actúan las diferentes fuerzas}

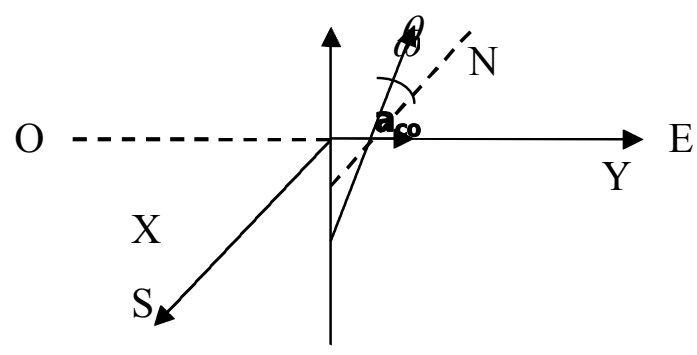

Figura 1. Dirección de la fuerza de coriolis.

- La fuerza de Coriolis actúa en todas las direcciones.

- Sobre el eje $Z$ actúa la gravedad, y el gradiente vertical de temperatura (variación de la temperatura con la altura) y la diferencia de presión.

- En todas las direcciones actúa la variación de energía 
Magnitudes

$\omega=7.292 \times 10^{-5} \mathrm{~s}^{-1}$ La velocidad angular de la tierra es relativa a las estrellas.

(la tienra gira en sentido anti-horario respecto al polo norte).

$\theta=45^{\circ}$ Grados latitud Norte. Ángulo formado respecto ala velocidad angular de la tienra y el eje norte-sur

$$
\begin{aligned}
& v=v \sin (\theta)=13.3797 \frac{\mathrm{m}}{\mathrm{s}} \text { La velocidad de la molécula de agua } \\
& m=\frac{\text { masa molar del agua }}{\text { númerode avogadro }}=2.9915887 \times 10^{23} \text { Masa de la molécula }
\end{aligned}
$$

\section{Fuerza centrífuga}

$$
\vec{F}_{\text {Centrifuga }}=-m \varpi \times(\vec{\omega} \times \vec{V})
$$

Aunque la fuerza centrífuga no la tenemos en cuenta porque es menor en tres órdenes de magnitud a la fuerza de Coriolis, explicamos su efecto en el huracán.

Cerca del centro del huracán, el viento es muy intenso, la fuerza centrífuga es la que equilibra a la fuerza del gradiente de presión (aunque la magnitud de la aceleracióncentrípeta es la misma tiene sentido contrario).

El gradiente de presión y la fuerza centrífuga forman un balance cidos tróficos.

Ojo del huracán

El ojo del huracán está modelado por medio de datos experimentales, tomados del huracán Isabel y del huracán Fabián.

En un huracán existe un área del aire que se orienta hacia el centro de este llamada ojo del huracán. En la zona baja el ojo posee temperaturas frías, contrario a lo que sucede en zonas altas, posee una geometría circular debido a que esta determina un estado de mínima energía.

La fuerza que describe el comportamiento físico del ojo del huracán es:

$$
f=v \frac{\partial p}{\partial r}
$$

Donde $v=\frac{4}{3} \pi r^{3}$ es el volumen de la gota de agua asumida como una esfera de radio uno milímetro $\mathbf{y} \frac{\partial p}{\partial r}=\mathbf{0 . 0 0 0 3 3}$ es la variación de la presión en función de la distancia al centro.

El valor de la variación de la presión se obtuvo por medio de datos experimentales realizados anteriormente, analizando graficas de diferentes huracanes y con su intensidad y tomando el valor mas general para la variación de presión comparando los datos obtenidos.

Variación de la Energía

Como el huracán se comporta como una especie de maquina de calor, en el cual se realiza un trabajo, podemos asociar el huracán con el cido de Camot adarando que el sistema tiene un cido irreversible, no ideal.

El cido se caracteriza por tener dos fuentes con una diferencia de temperatura, la de las gotas de agua en el mar y la de la troposfera, en donde el rendimiento es máximo, que trabaja 
absorbiendo una cantidad de calor $Q_{1}$ de la fuente de alta temperatura y cede calor $Q_{2}$ a la de baja temperatura produciendo un trabajo sobre el exterior (liberación de energía).

El rendimiento viene dado por:

En donde:

$$
\eta=\frac{W}{T_{1}}=\frac{T_{1}-T_{2}}{T_{1}}=\left(1-\frac{T_{2}}{T_{1}}\right)
$$

$T_{2}=198 \mathrm{~K}$ en la troposfera en donde se condensan las moléculas de gas (de agua) y

$T_{1}=300 \mathrm{~K}$ en el mar, es una condición inicial para que la formación de un huracán debido a que empiezan a evaporarse y ascender las moléculas de agua.

La fuerza que describe la variación de la energía es igual al calor latente por el número de gotas en la troposfera, por la eficiencia asociada al cido de Camot en la dirección de la velocidad, por una constante $K_{2}=3 \times 10^{-24}$ relacionada con la velocidad del huracán

$$
\frac{((1-\beta) \lambda n)}{V} k_{2}
$$

En la eauación 6 no consideramos la interacción moléaula-molécula.

Ecuación de movimiento

Finalmente la ecuación que describe la formación de un huracán es la suma de las fuerzas anteriormente descritas

$$
f=\frac{((1-\beta) \lambda * n)}{V}-2 m(\vec{\omega} \times \vec{V})+\left(-6.5 k_{1}\right)+V \frac{\partial p}{\partial r}
$$

Con $\lambda$ el calor latente del agua $\left(40.65 \mathrm{kj} \cdot \mathrm{mol}^{-1}\right)$ por número de moléculas condensadas $\mathrm{n}$ el número de partículas que se condensan en la troposfera (24660), v la velocidad de cada partícula $\beta=\frac{T_{2}}{T_{1}}$

El método utilizado para desarrollar las ecuaciones es el método de Verlet, se utilizo este método debido a que las ecuaciones no se podían resolver analíticamente, por tanto se hizo necesario usar programas como c++.

\section{Resultados}

El estudio se realizó en tres etapas analizando la formación del huracán por medio de la variación de temperatura en donde se incluye la variación de presión, la fuerza de Coriolis y finalmente la variación de energía. Para hallar la variación de temperatura recurrimos a datos experimentales.

\section{Análisis de gráficas}

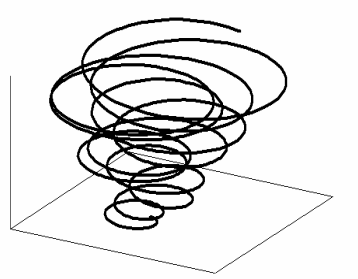

Figura 2. Formación de un huracán a una latitud de 45 grados. 
El sistema de coordenadas utilizado en la fig. 2 y en la fig. 3 es el de la fig. 1 con su respectiva orientación

La figura 2 nos ilustra la ecuación 6 y en ella podemos observar todo el proceso de formación de un huracán

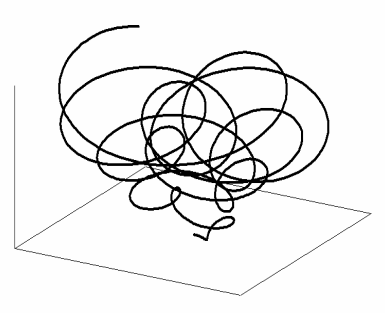

Figura 3. Formación de un huracán a una latitud de $\mathbf{1 5}$ grados.

La Figura 3. es una grafica que representa el proceso de formación de un huracán variando la latitud de $45^{\circ}$ a $15^{\circ}$ grados latitud norte.

Podemos analizar que en latitudes bajas no se produce un huracán y por lo contrario se produce un movimiento caótico, esto se explica por medio de la fuerza de coriolis debido a que esta no actúa en esta latitud y por tanto no aumenta la fuerza lo suficiente, ni dirige los vientos alrededor de un centro de baja presión para que se forme un huracán.

\section{Propuestas y Conclusiones}

La forma de modelar el fenómeno involucra diferentes fenómenos físicos: leyes de newton, fuerzas no inerciales, termodinámica, maquina de eficiencia.

Una propuesta para desarrollar es relacionar los efectos del cambio dimático con la formación de huracanes. Teniendo como marco de referencia las investigaciones realizadas por los científicos que han demostrado que el cambio climático no aumenta la formación de huracanes pero si aumenta su intensidad debido al calentamiento de la superficie del agua; se pueden induir otros factores que afectan en la formación de huracanes que no son mencionados en este artículo.

El modelo está abierto para ser reestructurado analizando condiciones iniciales como la temperatura pues éste no es sensible a ellas. También se puede generalizar utilizando un gran número de partículas en la formación del huracán teniendo en cuenta el comportamiento caótico de este.

Producciones desde los efectos de los huracanes

-Se puede aprovechar las características de un huracán para crear una planta de energía eléctrica debido a que al condensarse el vapor de agua se libera mucha energía que podría ser convertida en electricidad

Los huracanes abastecen de lluvia a los países calurosos que han sido afectados por sequías, de hecho muchas regiones deben sus cosechas a los huracanes.

\section{Bibliografía}

H. Goldstein. (1959). Mecánica Clásica. Addison Wesley

http: //uww.windows.ucar.edu/tour/link=/earth/Atmosphere/hurricane/eye.sp.htm/\&edu=high

Drifter Data in Hurricane Momentum Equation, during Fabian \& Jeanne (2003) and Frances\&Isabel (2004). http://umw.cora.nwra.com/ wmorzel/drifters. momentum.equation.html 